\section{Das geplante bundesdeutsche Erprobungsvorhaben zur heroingestützten Behandlung aus Sicht der teilnehmenden Städte}

Zusammenfassung: Nach gegenwärtigem Planungsstand werden sich die Studienorte des bevorstehenden bundesdeutschen Heroin-Erprobungsvorhabens auf bis zu acht Städte verteilen. Da sich die infrastrukturellen, epidemiologischen und fachpolitischen Vorbedingungen regional erheblich unterscheiden, stellt die Durchführung des Vorhabens nicht nur die einzelne Kommune vor gewaltige organisatorische, fachliche und finanzielle Herausforderungen. Auch der Städteverbund in seiner Gesamtheit wird permanent gefordert sein, der von ihm übernommenen Verantwortung trotz dieser Heterogenität möglichst konstant und konsensual nachzukommen. Die Städtekooperation wird hierbei getragen von dem gemeinsamen Willen, die aus kommunaler Sicht bislang als unzureichend erlebten (Be-)Handlungsoptionen im Umgang mit Opiatabhängigkeit wirkungsvoll zu erweitern. Dies kann nur gelingen, wenn das Vorhaben nicht allein an den arzneimittelrechtlichen Erfordernissen gemessen und ausschließlich als substanzbezogene Zulassungsstudie begriffen wird, sondern zugleich auch die medizinische Notwendigkeit, der Nutzen und die Wirtschaftlichkeit des dahinterstehenden Therapieansatzes validiert werden. Die an das Vorhaben geknüpften Erwartungen sind somit erklärtermaßen darauf gerichtet, das Outcome an Evidenzkriterien zu messen, die auch sozialversicherungsrechtlich operationalisierbar sein werden.

Schlüsselwörter: Opiatabhängigkeit - heroingestützte Behandlung - Städteverbund - Zulassungsstudie - Evidenzkriterien - Sozialversicherungsrecht

The Proposed German Multicenter Trial on Heroin-Assisted Maintenance Treatment in the View of Participating Cities: According to the current stage of planning the proposed multicenter trial on heroin assisted treatment (heroin maintenance) in Germany will be realized in up to eight cities. Since there are considerable differences between regions with regard to inescapable epidemiological, infrastructural and drug policy conditions, the project is a challenge for each participating municipality, demanding large financial and organisational efforts and treatment expertise. The board of participating cities will face the responsibility to deal with this heterogeneity in a consistent way and to achieve consent. Common purpose is an effective expansion of options for dealing with opiate dependency, since existing options so far have been felt as being insufficient. This idea can only succeed if the focus is not only on legal requirements for drug testing.

Suchttherapie 2000; 1: 67-70

(c) Georg Thieme Verlag Stuttgart · New York

ISSN 1439-9903
Peter Lindlahr*

Behörde für Arbeit, Gesundheit und Soziales, Hamburg
The project should not be understood as chiefly being a clinical test for substance approval. At the same time the treatment model behind should be evaluated, namely its necessity, advantage and good management. It is explicitly expected that outcome measurements should also yield evidence which is relevant to social insurance regulations.

Key Words: Opiate Dependency - Heroin Assisted Treatment - Multicenter Trial - Substance Approval - Outcome Measurements - Social Insurance Regulations

\section{Ausgangssituation}

Im Unterschied zur Schweiz, wo 1994 auf Initiative der dortigen Bundesregierung mit der PROVE-Studie zur modellhaften ärztlichen Verschreibung von Betäubungsmitteln begonnen wurde, ging hierzulande das Engagement zur Realisierung eines derartigen Ansatzes nicht von der Bundesebene, sondern von den Gesundheitsverwaltungen einzelner Länder und Kommunen aus.

Dementsprechend war schon zu Beginn der Neunzigerjahre in einigen bundesdeutschen Städten die gesundheitspolitische Erkenntnis gewachsen, dass zur qualifizierten ambulanten Behandlung Opiatabhängiger eine flächendeckende Ausweitung der Methadon-Substitution nicht ausreichen würde, um auf den Bedarf vor Ort angemessen reagieren zu können.

Parlamentarisch manifestiert wurde diese politische Überzeugungsbildung, als sich im Gesetzgebungsverfahren die Mehrheit der Bundesländer erstmals bereits im Juni 1993 [1] und sodann zu Beginn der darauf folgenden Legislaturperiode erneut im November 1994 [2] auf Antrag Hamburgs für die Möglichkeit aussprach, wissenschaftliche Erprobungsvorhaben zur heroingestützten Behandlung durchführen zu können. Bereits die damals angeführten Argumente zielten unmittelbar in Richtung der auch aktuell diskutierten [3] Outcome-Kriterien und des mit Heroin-Maintenance zu erreichenden Personenkreises. Es geht heute wie damals primär um die Reduzierung des Konsums von Straßenheroin, um gesundheitliche Stabilisierung sowie um individuelle Distanzierung von der Drogenszene bei denjenigen Opiatabhängigen, die mit Methadonbehandlung bisher nicht oder offenkundig schlecht erreicht wurden.

\footnotetext{
${ }^{*}$ Die Ausführungen geben ausschließlich die persönliche Meinung des Verfassers wieder und stellen keine dienstliche Äußerung dar.
} 
Diese Intention ist Ausdruck zweier synchroner, sich wechselseitig bedingender und bis heute gültiger drogenpolitischer Entwicklungslinien, die sich zum einen als Medizinalisierung, zum anderen als Kommunalisierung der Drogenpolitik begreifen lassen und jeweils eigenständige Prioritätensetzungen aufweisen.

Vor dem Hintergrund hoher drogeninduzierter Mortalitätsraten und steigender HCV-Prävalenz wurde in den vergangenen Jahren der Gedanke der harm reduction in weiten Teilen der Suchtkrankenhilfe für die Praxis operationalisiert und gilt dort längst als fester Bestandteil der jeweiligen Angebotssegmente. Zugleich engagierte sich das niedergelassene Vertragsarztsystem in zuvor nicht dagewesenem Umfang gegen teilweise erbitterten Widerstand der verfassten Ärzteschaft und des ärztlichen Establishments in der Substitutionsbehandlung Opiatabhängiger.

Folgerichtig verlagerten sich regionale und kommunale Akzentuierungen: Harm-reduction-Konzepte im Rahmen von niedrigschwelliger Kontakt- und Beratungsarbeit, Spritzentausch, Safer-Use-Training und die neuerdings durch das 3. Betäubungsmittelrechts-Änderungsgesetz [4] ausdrücklich legalisierte Konsumgewährung in hierzu zugelassenen Einrichtungen der Drogenhilfe bilden mittlerweile gemeinsam mit Substitution und komplementärer psychosozialer Betreuung Substituierter einen Schwerpunkt in den kommunalen und landesministeriellen Etats. Diese Form von Medizinalisierung der Drogenpolitik hat aber längst nicht nur bei den jeweiligen Veranschlagungen im öffentlichen Haushalt ihren Niederschlag gefunden. So deutlich wie nie zuvor offenbart etwa die Methadonsubstitution die Abhängigkeit der Politik und Gesundheitsverwaltung von selbstgesetzten, untergesetzlichen Normen der ärztlichen Selbstverwaltung [5], deren demokratische Legitimation momentan Gegenstand einer heftigen rechtspolitischen Kontroverse ist [6]. Wie nie zuvor ist deshalb drogenpolitischer Fortschritt vor Ort vom Verhandlungsergebnis mit Krankenkassen und verfasster Ärzteschaft abhängig, so etwa auch bei der ambulanten medikamentengestützten Detoxifikation oder bei der sich in der Behandlungspraxis zunehmend etablierenden Ohr-Akupunktur.

Auch die zweite Determinante, die Kommunalisierung bundesdeutscher Drogenpolitik, wirkt in vielfältiger Weise in das Handeln der vor Ort zuständigen Dienststellen hinein. Die innerstädtische Entstehung offener Drogenszenen in nahezu allen westdeutschen Großstädten, zugleich Ausdruck einer europaweiten Entwicklung [7], hat aufgrund der hiermit einhergehenden sozialen und gesundheitlichen Verelendungsprozesse nicht nur auf der klientenbezogenen Interventionsebene Handlungbedarf ausgelöst, sondern eine kritische Reflektion über die Entwicklung ganzer Stadtteile und der beträchtlichen Auswirkungen auf subjektive Sicherheitsbedürfnisse und Lebensqualität der dort ansässigen Wohnbevölkerung bewirkt. Insbesondere in großstädtischen Zentren wird Drogenpolitik zunehmend erlebt als Abwägungsprozess zwischen Interessen der Abhängigen, konzeptionellen Standortinteressen der Drogenhilfe, kommerziellen Standortinteressen der Gewerbetreibenden, logistischen Interessen der Sicherheitsbehörden und der Stadtplanung sowie elementaren lebensraumbezogenen Interessen der Anwohner. Wenn diese Gemengelage oftmals unter Anrufung der Gerichte [8], unter Zuhilfenahme professioneller Mediatoren [9] oder im Rah- men einer partiellen Neubestimmung des polizeilichen Präventions-(Gefahrenabwehr-)Verständnisses aufzulösen versucht wird, fällt auf, wie diffus und fragil die öffentlichen Erwartungen an eine modellhafte heroingestützte Behandlung derzeit noch sind. Insofern erfordern, jenseits aller methodisch-wissenschaftlichen, rechtlichen und finanziellen Rahmenbedingungen auch die widerstreitenden kommunalen Interessenlagen vor Ort, dass die bevorstehende Durchführung des Vorhabens durch Bund, Länder und Städte möglichst konsistent realisiert und frühzeitig für eine qualifizierte kommunikative Begleitung in den regionalen und kommunalen $\mathrm{Zu}-$ sammenhängen gesorgt wird.

\section{Strukturelle Prämissen bei der Durchführung des Modellvorhabens}

Die bevorstehende Durchführung des Modellvorhabens ist in den beteiligten Städten und Ländern über Jahre hinweg reflektiert und einer fachpolitischen und finanziellen Folgenabschätzung unterzogen worden. Die „Arbeitsgemeinschaft der Großstädte zur Drogenpolitik“ war ebenso mit dem Thema befasst wie der Deutsche Städtetag, dessen Gesundheitsausschuss in seiner 114. Sitzung am 15./16. Januar 1998 in Halle/ Saale die Autonomie der betroffenen Kommunen grundlegend bekräftigte. Im dortigen Beschluss heißt es u.a.: „Der Gesundheitsausschuss des deutschen Städtetages ist der Auffassung, dass Städte, die die modellhafte Erprobung ärztlich kontrollierter Abgabe von Heroin an Schwerstabhängige für erforderlich halten, die Freiheit haben, eine solche Entscheidung zu treffen.“[10]

Von der so zugestandenen „Freiheit“ werden voraussichtlich die Städte München, Karlsruhe, Frankfurt am Main, Köln, Essen, Hannover, Hamburg und nach neueren Entwicklungen wohl auch Berlin Gebrauch machen.

Hierbei werden die Kommunen in den Flächenländern nur in Niedersachsen, Nordrhein-Westfalen und Hessen mit politischer und finanzieller Unterstützung ihrer jeweiligen Landesregierungen rechnen können. München und Karlsruhe beteiligen sich trotz ausdrücklicher Ablehnung der bayerischen Staatsregierung und der baden-württembergischen Landesregierung. So unterschiedlich die politische Willensbildung vor Ort und damit auch die Bewertung fachpolitischer Fragen zwischen den Partnern des Städteverbundes ausfällt, so heterogen sind auch die fachlichen und infrastrukturellen Voraussetzungen in den jeweiligen Regionen.

Großstädtische Drogenszenen wie in Hamburg oder Frankfurt weisen nicht nur tendenziell andere Entwicklungen, Wechselwirkungen und Konsumprävalenzen auf, als dies bei kleineren Städten der Fall ist. Auch die Infrastruktur der Hilfeangebote, das fachbehördliche Monitoring des lokalen Hilfesystems [11] und die logistischen Möglichkeiten bei der Vernetzung von Hilfen bewegen sich in einem anderen Rahmen, als dies in einer kleineren Stadt möglich wäre. Dementsprechend divergiert auch ganz erheblich der örtliche finanzielle Rahmen, innerhalb dessen die Städte jeweils ihre drogenpolitischen Maßnahmen realisieren (müssen).

Angesichts dieser Heterogenität erlangt der Umstand besondere Bedeutung, dass für die bevorstehende Studie ein die Rahmenbedingungen mitgestaltender gewerblicher Sponsor, 
im Bereich der klinischen Arzneimittelprüfung üblicherweise der pharmazeutische Unternehmer, gänzlich fehlt. Dieser eher atypische Sachverhalt hat die Diskussion zwischen Bund, Ländern und Städten darauf gelenkt, die Interessenlage des Bund-Länder-Städte-Verbundes als öffentlicher Sponsor stärker als in der bisherigen Diskussion zu differenzieren. Danach bleibt es zwar dabei, die substanzbezogene Arzneimittelprüfung als konstitutives Element der Zulassungsstudie mit aller rechtlich und wissenschaftlich-methodisch gebotenen Sorgfalt zu veranlassen und durchzuführen. Allen Beteiligten ist zugleich jedoch bewusst, dass das Modellvorhaben in seiner gesamten Konfiguration und dem hierfür veranschlagten zeitlichen Umfang aber über eine bloße Arzneimittelprüfung deutlich hinausgeht.

In der Bekanntmachung des Bundesministeriums für Gesundheit vom 17.9.1999 [12] ist als „Zielsetzung“ des wissenschaftlichen Vorhabens ausdrücklich formuliert, dass

- die zu konzipierende Studie die klinische Prüfung heroinhaltiger Arzneimittel beinhalten solle (Zulassungsstudie)

- „sowie einen zusätzlichen Erkenntnisgewinn u.a. zu der Frage erbringen“ soll, „ob und wie (...) die heroingestützte Behandlung in das Therapieangebot zur Versorgung Opiatabhängiger implementiert werden kann“.

Dem geplanten klinischen Erprobungsvorhaben liegt somit ein zweifaches wissenschaftliches Erkenntnisinteresse zugrunde. Es geht sowohl um die substanzbezogene Prüfung, insbesondere die pharmakologisch-toxikologische Prüfung im Sinne des $\S 40$ Absatz 1 Nr.6 AMG. Die substanzbezogene Prüfung ist zwar auf ein definiertes Indikationsgebiet gerichtet. Die hiermit verbundenen Behandlungsaspekte betreffen jedoch in einem ausschließlich arzneimittelrechtlichen Verständnis lediglich den bestimmungsgemäßen Gebrauch der Substanz im Sinne des Arzneimittelgesetzes und nicht etwa die Wirksamkeit und Wirtschaftlichkeit des therapeutischen Behandlungsansatzes im Sinne des SGB V.

Deshalb muss das wissenschaftliche Interesse zugleich gerichtet sein auf die behandlungsbezogene Prüfung. Die Implementierung bislang gar nicht oder nicht als Standardtherapie angewendeter Behandlungsmethoden in das medizinische Versorgungsangebot erfolgt nach den Vorgaben des SGB V. Der Gesetzgeber hat hierbei die Normsetzung durch untergesetzliche Regelungen der ärztlichen Selbstverwaltung zugelassen und insoweit den Bundesausschuss der Ärzte und Krankenkassen (BÄuK) mit einer umfassenden Normsetzungsbefugnis ausgestattet. Um ein einheitliches Vorgehen bei der Implementierung neuerer Behandlungsansätze zu ermöglichen, hat der BÄuK erstmals nach Inkrafttreten des 2. NOG-GKV Ende 1997 [13] allgemeingültige Kriterien und die hierauf bezogenen Bewertungsverfahren festgesetzt. Am 10.12.1999 [14] hat er diese fortan als „BUB-Richtlinien“ bezeichnete Bewertungsrichtlinie aktualisiert. Die Bewertung einer Methode ist danach grundsätzlich an ihrem Nutzen, ihrer medizinischen Notwendigkeit und ihrer Wirtschaftlichkeit zu orientieren.

Um eine valide Bewertung vornehmen zu können, bedarf es im Einzelnen definierter Nachweise in den vom BÄuK festgesetzten unterschiedlichen Evidenzstufen bzw. Evidenzklassen. Der Nutzen einer neu eingeführten Behandlungsmethode ist nach Nr. 6.4. der o.g. Richtlinie durch mindestens eine ran- domisierte, kontrollierte und nach international anerkannten Standards (GCP, Consort) durchgeführte und veröffentlichte Studie zu belegen.

Damit muss das geplante Vorhaben zur Beurteilung des Nutzens des Therapieansatzes über eine reine prospektive Interventionsstudie (BUB-Richtlinie, Nr. 8.1, Evidenzstufe II a) hinausgehen und sich an den Standards der Evidenzstufe I orientieren. Untersucht wird hier also der Interventionsansatz („treatment“) in Bezug auf sämtliche der in der BMGBekanntmachung unter „Zielsetzung“ benannten Parameter.

Die Partner der Koordinierungsgruppe (Bund, Städte, Länder) gehen davon aus, dass beide Untersuchungsgegenstände parallel zu überprüfen sind, hierbei jedoch unterschiedliche Untersuchungszeiträume erforderlich sein werden. So könnte insbesondere bei Heranziehung und vergleichender Auswertung der in der Schweiz vorliegenden sog. „RegistrierungsDossiers“ - die substanzbezogene Prüfung einen erkennbar geringeren zeitlichen Aufwand beanspruchen als die behandlungsbezogene Prüfung. Einzelheiten hierzu werden durch die im Prüfplan definierten Outcome-Kriterien, durch die hierauf bezogene Stichprobengröße und durch die jeweiligen Erhebungszeitpunkte für den individuellen Verlaufsstatus bestimmt. Anhand dieser Vorgaben ist für die noch ausstehende bundesbehördliche betäubungsmittelrechtliche Ausnahmegenehmigung die Frage zu diskutieren, ob für die vorliegende Studienkonzeption das vom Gesetzgeber in $\S 3$ Absatz 2 BtMG beschriebene Tatbestandsmerkmal „zu wissenschaftlichen (Zwecken)“ als Erlaubnisgrundlage ausreicht oder ob die Beantragung der behandlungsbezogenen Prüfung auch (ggf. hilfsweise) auf den Erlaubnistatbestand „(zu) anderen im öffentlichen Interesse liegenden Zwecken“ gestützt werden muss. Die Verfügbarkeit der Prüfarznei muss aus Sicht der Städte auch dann sichergestellt sein, wenn die substanzbezogene Prüfung („Zulassungsstudie“) bereits abgeschlossen ist und das Zulassungsverfahren betrieben wird. Wegen des einheitlichen Prüfplans wird es sich aber nicht um eine eigenständige Follow-up-Studie im herkömmlichen Verständnis handeln. Vielmehr wird die in die Gesamtstudie integrierte Arzneimittelprüfung bereits zum Abschluss gelangt sein. Für diese Phase wird die betäubungsmittelrechtliche Erlaubnis allein auf die (noch andauernde) behandlungsbezogene Prüfung gestützt werden können. Der formale Einwand, es handele sich dann faktisch um eine Interventionsstudie mit einer arzneimittelrechtlich nicht zugelassenen Substanz, trifft nicht $\mathrm{zu}$, da die formellen und materiellen Voraussetzungen für die Zulassung durch die zuvor abgeschlossene klinische Prüfung ja bereits geschaffen sind und lediglich der prozessuale Aufwand (Zulassungsverfahren) weitere Zeit beansprucht.

Die strikte Orientierung an den nach GCP unerlässlichen Standards stellt Bund, Länder und Städte gleichermaßen vor die Herausforderung, das Modellvorhaben im Sinne eines gemeinsamen Sponsorships zu tragen und hierbei nicht nur untereinander, sondern auch für den mit der Konzeption und wissenschaftlichen Durchführung beauftragten Studienleiter die im Verlauf der Studie vorzunehmenden Operationalisierungen, Entscheidungs- und Abstimmungsprozesse mit einem hohem Maß an Transparenz zu versehen.

Vor diesem Hintergrund ist zwischen Bund, Ländern und Städten ein öffentlich-rechtlicher Kontrakt erarbeitet worden, 
dessen Unterzeichnung vor Erteilung des Forschungsauftrags vorgesehen ist und der gewissermaßen als „Geschäftsgrundlage“ zwischen den Beteiligten dient. Diese Vereinbarung sieht vor, dass

- die notwendige Differenzierung zwischen Arzneimittelprüfung und Therapiestudie vorgenommen wird,

- die Entscheidungsprozesse während des Vorhabens durch eine gemeinsame Lenkungsgruppe zentral gesteuert werden,

- die Differenzierung der Kosten auf eine dezidierte schriftliche Vereinbarungsgrundlage gestellt wird,

- die Bindung der Beteiligten an das Vorhaben für unabdingbar erklärt wird und bei Gesamtbeendigung oder unvorhergesehenem Ausscheiden Einzelner eine qualitativ hochwertige Anschlussbehandlung für die hiervon betroffenen Probanden sichergestellt wird.

\section{Erwartungen an das drogenpolitische Outcome des Modellvorhabens}

Die an das Modellvorhaben geknüpften Erwartungen werden selbst über die Parteigrenzen hinweg in der politischen Diskussion allzu oft mit einer Verringerung des drogenbedingten innerstädtischen Problemdrucks assoziiert. So postulierte schon im eingangs erwähnten Gesetzgebungsverfahren die damalige Regierungsfraktion der FPD: „Insbesondere die innerstädtischen Ballungsräume brauchen diese Form der Hilfe“ [15]. Die gegenwärtige Drogenbeauftragte der Bundesregierung geht noch weiter, wenn sie öffentlich erklärt, von dem geplanten Modellvorhaben erwarte sie „eine höhere Lebensqualität für die Bevölkerung in jenen Gegenden, die zuvor Schauplatz der Drogenszene gewesen seien“ [16].

Es dürfte unbestritten sein, dass auch in den beteiligten Kommunen angestrebt wird, derart hypothetische Kausalitäten eines Tages verifizieren zu können.

Bis dahin begnügt man sich mit dem hohen Anspruch, innerhalb der kommenden drei Jahre die Voraussetzungen für die Einleitung des arzneimittelrechtlichen Zulassungsverfahrens und eine valide Erkenntnisgrundlage für die Gremien der gesetzlichen Krankenversicherung zur dortigen Bewertung des Nutzens, der medizinischen Notwendigkeit und der Wirtschaftlichkeit des Behandlungsansatzes Diamorphin Maintenance Treatment erlangt zu haben.

\section{Literatur}

${ }^{1}$ Drucksache 12/5673. Deutscher Bundestag

2 Drucksache 13/205. Deutscher Bundestag

${ }^{3}$ So im Wesentlichen die auf die Bekanntmachung des BMG im BAnz. Nr.183 vom 29.9.1999 eingereichten Studiendesigns: Gastpar M. Wissenschaftliche Erprobung der ärztlichen Heroinverschreibung; Krausz M. Das bundesdeutsche Modellprojekt zur heroingestützten Behandlung Opiatabhängiger - eine randomisierte Kontrollgruppenstudie, Dezember 1999 (beide unveröffentlicht)

${ }^{4}$ Drittes Gesetz zur Änderung des Betäubungsmittelgesetzes. vom 28.3.2000, BGBl. I, 302 ff.

${ }^{5}$ Bekanntmachung des Bundesausschusses der Ärzte und Krankenkassen über die Neufassung der Richtlinien über die Einführung neuer Untersuchungs- und Behandlungsmethoden und über die Überprüfung erbrachter vertragsärztlicher Leistungen („BUB-Richtlinien“). vom 10.12.1999, BAnz. Nr.56 vom 21.3.2000, $4602 \mathrm{ff}$.

${ }^{6}$ Bundessozialgericht, Urteil vom 16.9.1997 1 RK 28/95 In: BSGE 81, 54 ff.; Di Fabio U. Verlust der Steuerungskraft klassischer Rechtsquellen. Neue Zeitschrift für Sozialrecht (NZS) 1998; 449 ff.; Oldiges FJ. Bundesausschuss der Ärzte und Krankenkassen: ein neues Machtzentrum in der GKV?. Die Ortskrankenkasse 1997, 367 ff.; Schimmelpfennig-Schütte R. Richtliniengebung durch den Bundesausschuss der Ärzte und Krankenkassen und demokratische Legitimation. NZS 1999, 530 ff.; Wimmer R. Grenzen der Regelungsbefugnis in der vertragsärztlichen Selbstverwaltung. NZS 1999, $113 \mathrm{ff}$.

${ }^{7}$ Lange KJ, Renn H. Urban Districts and Drug Scenes, A comparative study on nuisance caused by open drug scenes in major european cities. 1995

${ }^{8}$ Bundesgerichtshof, Mitteilung der Pressestelle vom 7.4.2000 („Frankfurter Drogenhilfezentrum“) zum Urteil des V. Zivilsenats -V ZR 39/99 -, das die Anwohnerklage gegen eine sog. „niedrigschwellige“ Drogenhilfeeinrichtung zurückwies

${ }^{9}$ Gessenharter W. Mediatorenverfahren „Weitere Gesundheitsräume in St. Georg? Wünschbarkeit, Zweckmäßigkeit, Notwendigkeit“. Abschlussbericht und Empfehlung des Mediators an den Senat der Freien und Hansestadt Hamburg, Juli, 1999

${ }^{10}$ Deutscher Städtetag, Vorbericht für die 115. Sitzung des Gesundheitsausschusses vom 18.5.1998

11 Schmid M, Simmedinger R, Vogt I, BADO e.V. Ambulante Suchthilfe in Hamburg, Statusbericht 1998 zur Hamburger Basisdatendokumentation im ambulanten Suchthilfesystem. ISS-Schriftenreihe, Frankfurt: 1999

12 Bundesanzeiger. vom 29.9.1999, 183: $16753 \mathrm{ff}$.

13 Richtlinien vom 1.10.97, veröffentlicht im Bundesanzeiger vom 31.12.1997, 243: 15232

14 Bundesanzeiger vom 21.3.2000, 56: 4602

${ }^{15}$ Leutheusser-Schnarrenberger S, Thomae D. Neue Wege in der Drogenpolitik beschreiten. Pressedienst der FDP-Bundestagsfraktion vom 19.6.1997

16 „Nickels wirbt für Modellversuche“. FAZ vom 16.2.2000
Peter Lindlahr

Tesdorpfstraße 8

20148 Hamburg 